\title{
An approach for 3D visualization of pipelines
}

\author{
Y. Du and S. Zlatanova
}

The urban planning bureau of Yibing city, Sichuan Province, P.R.China GISt, OTB, Delft University of Technology, Delft, The Netherlands

\begin{abstract}
Utility networks (pipelines and cables) are usually modelled by lines and nodes. The systems used for maintenance of utilities are typically twodimensional, allowing for creating vertical profiles in limited key places. Since the position is visualized on 2D maps, it is rather difficult to distinguish between cables and pipelines with the same $\mathrm{x}, \mathrm{y}$ coordinates. Such visualization might be misunderstood by both field workers (maintaining the utilities) and specialists. Therefore the interest in $3 \mathrm{D}$ visualization is rapidly increasing.

Generally, visualization of lines in 3D is quite challenging. In most of the cases, the best solution is to replace the lines with tiny cylinders or other 3D shapes. However, substituting simple lines with parametric shapes in the database is time consuming, expensive and economically not justified. This paper presents an approach for utility management, according to which the pipelines and cables are still maintained as lines but while visualising in $3 \mathrm{D}$, the lines are substituted with cylinders created on the fly.

This paper presents the used systems architecture and developed prototype. A final discussion summarises on advantages and disadvantages of the used approach and outlines directions for further research.
\end{abstract}

\section{Introduction}

Visualisation of utility networks has always been problematic. Utility networks are usually represented as lines (segments of the networks) and 
points (connections, valves, etc.) predominantly with their $\mathrm{x}, \mathrm{y}$ coordinates. Depending on the type of the utility networks (water, swage, telecom, etc.), the depth or (more rarely) the $\mathrm{z}$ coordinates (in given points) might be registered. The software (GIS, CAD, AM/FM) for utility maintenance is typically 2D, i.e. the visualisation of all the elements of the networks is on 2D maps (Figure 1). Such a visualisation usually serves the needs of a company (or state authority) that is responsible for a particular network, but can result in misinterpretation when provided to third parties. Various factors contribute to confusion and misinterpretation of the information on 2D maps. Firstly, the major trace of pipelines or cables per network is mostly the same, i.e. under the streets, which results in overlapping lines on the map. To avoid this overlap, many companies offset the multiple pipelines to increase the readability of the map. Such an approach, however, could mislead unfamiliar users. Secondly, the trace of the different utility networks also overlaps. Colour and depth (depicted near a segment) of a particular pipe or cable are often the only parameters to distinguish between different networks. Integrating several networks on one map is almost an impossible task. Thirdly, a large number of important elements of the networks (such as valves, connections) are given with symbols, which might be challenging for interpretation from non-specialist and even from some of the less-qualified field workers. Finally, some of the networks (e.g. sewage) contain a large amount of vertical elements, which visualisation on the 2D maps is only as points. Explanations about the vertical elements is often not included in the maps relying on the on-site experience of field workers

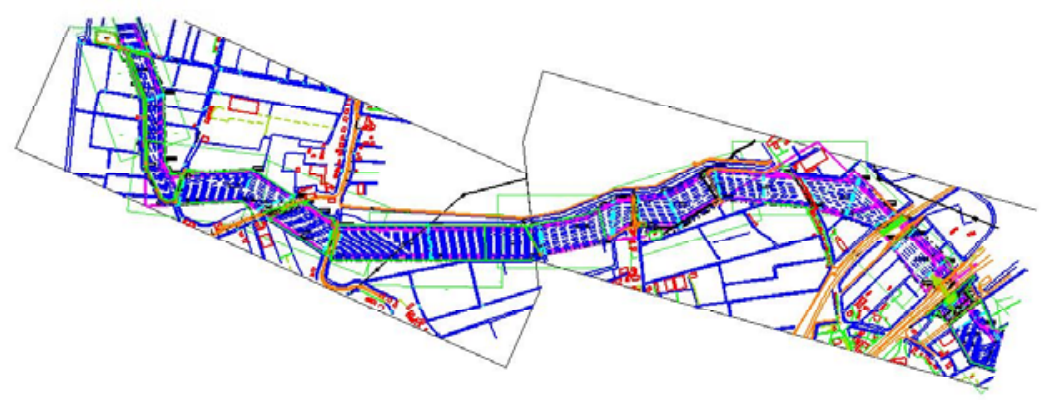

Figure 1: Visualization of Utilities on a 2D map

These drawbacks of 2D visualization of utilities are not new. However, the rapidly increased utilization of underground space by utility companies requires more extended knowledge about the position of underground utility networks that ever before. The intensive expansion and modernization of cities (involving re-construction of streets, buildings etc.) needs reliable 
information about existing infrastructures. Resent investigations (Roberts et al 2002) have revealed an increased number of accidents of various ranges and scales. It is clear to many governments that a 'centralized management' of utilities is the only way to improve the knowledge on the underground infrastructure (Chong 2006, Hei et al 2002, Penninga and Oosterom 2006). Among all the aspects related to a centralized registration such as appropriate system architecture, type of information to be stored, legislation, etc., is also the visualization approach.

3D visualization of utilities is considered by many (Zlatanova 2004, Du 2005, Chong 2006) as able to solve many of the drawbacks mentioned above. 3D visualization of pipelines is necessary tendency for development of urban pipe and cable systems, because it can clearly express the position and spatial relationship of all pipelines. Arbitrary displays of pipelines from any view and/or from any a place (also for profiles) can be created. In such a way, blind-cutting and fault damaging of pipelines can be considerably avoided. Particularly, in the case of crisis recovery after unexpected accidents or natural disasters, e.g. emergent fires, gas-leakage, anti-terrorism, flooding and earthquake, 3D visualization may provide vivid graphics in such a crisis epoch for a quick decision-making, in order to save precious time and to avoid life and economical loss as soon as possible.

Research is emerging aiming at improved utility visualization. Roberts et al 2002 suggest an augmented reality system for 3D visualization of utilities (showing their position on the surface with attached depth information). Peng et al 2002 discuss profile creation from a utility model to maintain the pipes and the lines with their 3D coordinates. He et al 2002 present a formal approach for underground utility system.

This paper concentrates on 3D visualization created on the fly from utility records maintained in DBMS. Next section discusses possibilities for realizing such a concept and gives a motivation for the selected system architecture. Section 3 and section 4 elaborate on developments in detail. The last section provides an extended analysis on the obtained research and outlines directions for further research.

\section{System Architecture for management and 3D visualization of utilities}

Currently, there are numerous approaches to implement 3D visualization for pipelines. First, it could be only for the purpose of visualization, i.e. one can execute algorithms or functions of CAD (Computer-Aided De- 
sign) to draw 3D figure with OpenGL or Direct3D language (e.g. Yong 2003). However, this may not fully make use of semantic (attribute) information. The second approach is based on CAD software packages for modelling, visualization and, quite often, some network analysis specific for a particular utility network. Such systems, known as automated mapping and facility management (AM/FM) were extensively developed in early 80 's and some of them provided even 3D visualization. Many originally CAD vendors nowadays provide extensions to their software that deal with utilities. These systems provide reach tools for 3D visualization but for a specific utility network. The third approach is based on 2D GIS, $3 \mathrm{D}$ visualization packages are provided by most of traditional GIS vendors, such as 3D Analyst of ArcGIS, GeoMedia Terrain, and so on. Whereas, they do not provide a solution for 3D modelling of pipeline.

Spatial DBMS is yet another possibility to organize information of utilities. The advantages of a central spatial DBMS is apparent: spatial and attribute data are maintained in one integrated environment (Oosterom et al 2002). Data in spatial DBMS can be currently accessed by most of $\mathrm{CAD} / \mathrm{GIS}$ system, which is yet another very promising solution to $3 \mathrm{D}$ visualization (Zlatanova et al 2002, Zlatanova and Stoter, 2006).

Among all the approaches, this paper gives preferences to the last one motivated by the strong management characteristics of DBMS and 3D editing/visualisation possibilities of CAD software. On the one hand, there are many convincing reasons to use Spatial DBMS for management of utility data sets: multi-user control on shared data and crash recovery, automatic locks of single objects while using database transactions, advanced database protocol mechanisms to prevent the loss of data, data security, data integrity and operations that comfortably retrieve, insert and update data. On the other hand, CAD systems provide flexible tools for 3D editing, adaptable, user-friendly graphic user interfaces, power means for realistic rendering and navigation through $3 \mathrm{D}$ models, possibilities to create animations, different views and export data in various formats (Breunig and Zlatanova, 2006). Therefore, in our approach spatial DBMS is used to manage the utility networks (with their 3D coordinates but still organised as lines and points) and CAD software is used for the $3 \mathrm{D}$ visualisation, as the $3 \mathrm{D}$ shapes are created on the fly.

\section{From 2D to 3D}

In this paper we concentrate on the visualization of pipeline networks since their complexity is a bit higher compared to cables. However, the as- 
sumptions bellow can be easily extended to cable networks. Compared to other topographic data, pipelines and cables are relatively simple types of data. Pipelines are generally represented by the $x, y$ coordinates of their central lines. Depth or z-coordinate is not a compulsory parameter for all networks. For example, the water company Waterleidingbedrijf Amsterdam responsible for drink water in Amsterdam, the Netherlands does not maintain any of them. In contrast, the urban offices in China have records for both depth and z-coordinate. Pipelines are mostly straight lines with relatively small number of turns. Very often the turns of the pipes are represented in 2D maps with one point. Relatively large turns are currently presented using short straight lines connecting successive interpolation points. The interpolation points of arc are required to be within a certain precision.

The accuracy of points within the pipeline networks is relatively high. The horizontal precision is around $\pm(5+0.08 \mathrm{~d}) \mathrm{cm}$, and the vertical precision is within $\pm(5+0.12 \mathrm{~d}) \mathrm{cm}, \mathrm{d}$ is the depth of the centre of pipeline. Shape and size of pipelines are relatively consistent, and materials of pipelines are rather limited. Joints of pipelines are almost the same for a single pipeline. The shape of the most pipelines is cylinder though some of them are ditch.

As already mentioned above the vertical segments in current networks are either not represented or indicated implicitly with symbols and textual information. Accesses from the surface are also defined only by symbols.

These properties of pipelines make the visualization of pipelines in 3D quire straightforward and easy. Long straight lines can be replaced with tiny cylinders considering the diameter of the pipe and consequently visualized as $3 \mathrm{D}$ objects. The real challenge is the missing information:

- absence of vertical segments,

- visualization of turns and intersection of cylindrical pipes,

- re-construction of rectangular pipes,

- 3D symbols to replace the 2D symbols.

\section{Absence of vertical elements}

Originally, the networks contain (in most of the cases) only the horizontal segments. In case of different depth, double depth values are recorded by the end points of the segments. Figure 2 illustrates the creation of the vertical elements. In the given example, the original pipe consists of only four segments represented by 5 points (Figure 2, above). These segments are further extended with two more points (6 and 7) and two more corresponding vertical segments (Figure 2, bellow). Clearly, if the depth (z- 
coordinate) is not available it should be measured. Measuring methods to obtain the exact position of the underground utility networks is discussed in $\mathrm{Du}, 2005$.

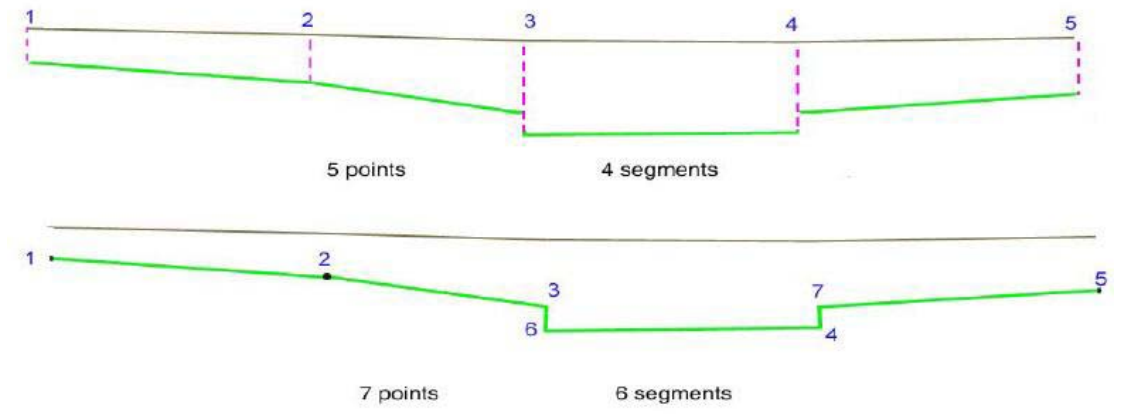

Figure 2: Transition from 2D data set to 3D: creation of vertical segments

Visualization of turns and intersections of cylindrical pipelines

Reconstruction of the shape of cylindrical pipelines is relatively simple. Cylindrical pipelines are practically composed of many cylinders taking into consideration the value of diameter and coordinates obtained from centre lines. In case the pipe has a variable diameter, the shape to be created is a cone. Since these cylinders are all straight, gaps and superimposition appear at the joint between two segments (Figure 3). It is apparent, the larger changes in the directions of the pipes, the more visible discrepancies would appear. The size of gap may change also with respect to the diameter of the pipe. The best way to avoid such gaps is to use smooth transition from one pipe to another considering the pipeline radius (Figure 4). Such a transition can be realized by introducing a new curve segment (e.g. torus) between the two pipes. However, this approach may require quite significant changes in the original data. The torus should be created with a certain offset depending on the diameter of the pipes and the angle between the central lines. Since the complexity of the computation increases, such a solution might result in dropping down the performance.

Another option is the display a sphere at the joints of any successive segments. The radius of the sphere is to be determined with respect to the radius of the two connecting pipes. An advantage of this approach is that sphere is the simplest surface and does not depend on the rotation matrix in 3D space (needed to adjust a shape in the 3D scene). Although we have not tested the influence of adding spheres in all connections for very large data sets, we expect that the performance will not be violated. The 3D visualization on-fly procedure includes the following steps: 
- Query the lines and the points to be visualised in 3D from the DBMS (get the 3D coordinates)

- Query the diameter of the pipe segments

- Construct a cylindrical segment (or a cone element)

- Construct a sphere joint

- Display in the view windows of a CAD


(1) 2D planar graphics (Red is central line, Green is angle bisector)
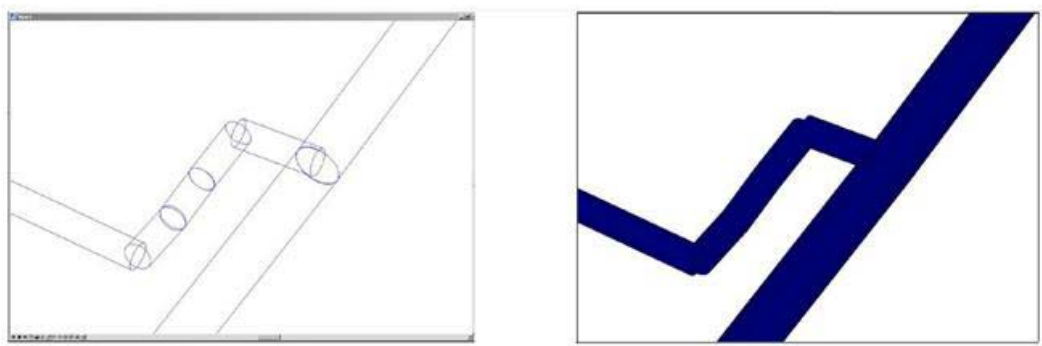

(2) 3D visualization

Figure 3: 3D visualization of cylindrical pipes without connections

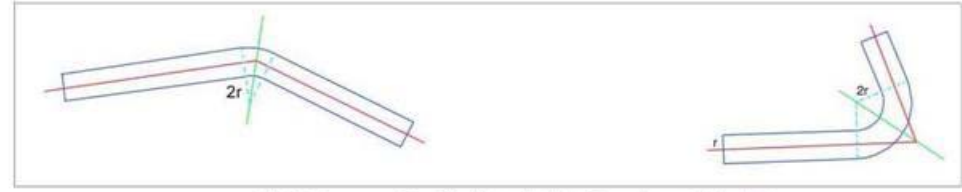

(1) $2 \mathrm{D}$ planar graphics (Red is central line, Green is angle bisector)

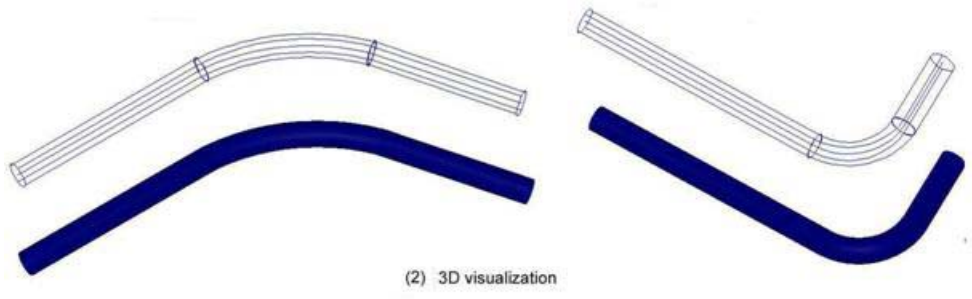

Figure 4: 3D visualization of cylindrical pipes with smooth transition using torus . 


\section{D visualization rectangular pipes}

Rectangular pipes are mainly ditches and those containing cables and groups of cables of telecom and electricity companies. The containers are defined by the Minimum Bounding Cuboid (MBC) that minimally encloses the cables. 3D visualization of rectangular pipelines is carried out by simulating a hexahedron, which is constructed by eight vertices and four faces. The rectangular shape obeys two restrictions: for planarity and verticality. In order to keep the visual impression undisturbed, the faces (composed of four vertices) are considered planar. Having in mind the shape of rectangular pipelines in reality, the two side faces (right- and lefthand) are restricted to be vertical.

The computation of vertices of the hexahedron is the critical step of visualization of rectangular pipelines. This paper proposed a bit simplified computational approach. The coordinates of the vertices are determined by the coordinates of the central line and the size of the pipeline. Firstly, a vertical section is made through the central line. In the vertical section, two lines parallel to the central line are constructed, by both sides respectively. The distance to the central line is half of pipelines size, see Figure 5. The cross points of parallel lines with the transects of two ends in AB segment are presented as point a1, a $2, \mathrm{~b} 3$ and $\mathrm{b} 4$. Whereas, there may be 4 points in about one central point of pipeline, i.e. $\mathrm{b} 1, \mathrm{~b} 2, \mathrm{~b} 3$ and $\mathrm{b} 4$ in point $\mathrm{B}$. The coordinates of a1,a2,b3 and b4 then can be easily computed (see Du, 2005).

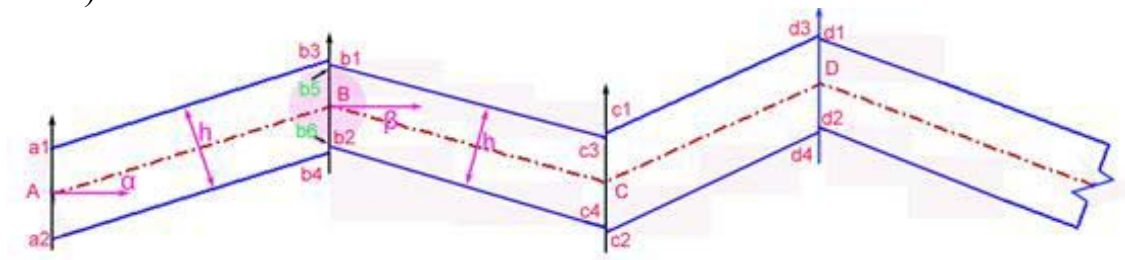

Figure 5: Computation of rectangular pipes

It should be noticed that only if the inclination angles ( $\alpha$ and $\beta$ ) of the forward and afterward segments are equal, point $b 1$ and point $b 3$, and $b 2$ and $\mathrm{b} 4$ are same points. Otherwise there are always 4 points (only $\mathrm{Z}$ is different) at any middle vertex of central line of pipeline. This means that there is a sudden change in the heights. Because the angles are rather small, we have decided to considered these points equal. Point b1 and point $\mathrm{b} 3$ are moved to the location of point $\mathrm{b} 5$, and point $\mathrm{b} 2$ and $\mathrm{b} 4$ moved to the location of point b6. Applying this simplification, we ignore the small gaps between $\mathrm{b} 1-\mathrm{b} 3$ and $\mathrm{b} 2-\mathrm{b} 4$ to ensure the $3 \mathrm{D}$ visualization. The 
disadvantage is that the height of the pipelines may be changed though the change is rather small.

We have investigated all possible changes in the direction of the pipes and we have concluded that there are four distinct cases possible (Figure 6, :

- Vertices of the beginning (or ending) pipeline, see location A,

- Vertical middle vertices (only $\mathrm{Z}$ is different), location $\mathrm{BC}$,

- Middle vertices, see location D,

- Vertical ending vertices, see location EF.

It should be noticed that the direction of the consequent segment (up or down $\backslash$ ) is not of importance. Cases A and EF differ from respectively D and $\mathrm{BC}$ in the closing faces. Detailed formulas regarding the computations in each particular case are given in $\mathrm{Du}, 2005$.Figure 7 illustrates the obtained results.

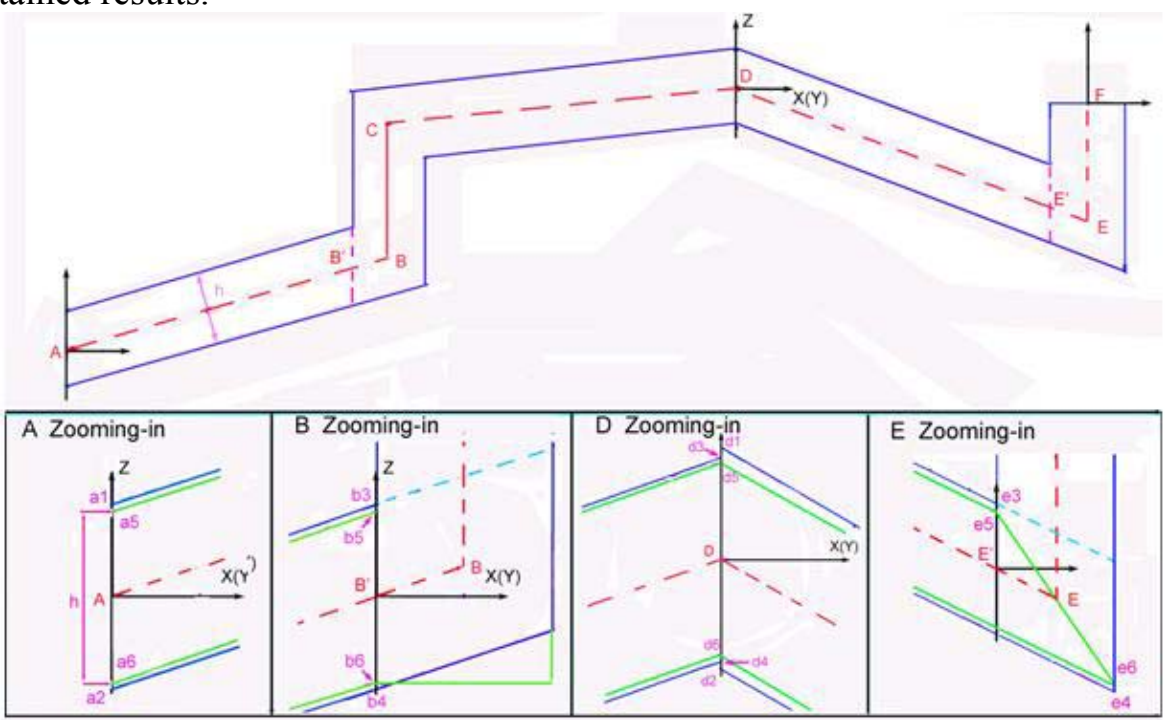

Figure 6: Four different cases of pipe changes

\section{D symbols}

Usually, 3D symbols can be easily created in any CAD program, stored in a uniform library and used when necessary. A symbol is made up of series of graphic elements. Each symbol has an origin, which is defined when the cell is created. For this study, we have created only few basic 3D symbols, i.e. valve, hydrant, well for check, man-hole, hand-hole and control box (Figure 8). One can update and/or append a symbol at any time. 
The steps to follow are almost similar for all CAD programs. Bellow the procedure to create symbol library in Microstation is listed:

- Attach a file containing a cell library (if no cell library, create new one).

- Draw the contents of the cell on the desired levels.

- Select all the elements to be included in the cell with the Element Selection tool or fence tool.

- Define Cell Origin tool. The cell origin is the point that is used to position the cell in a design. When a cell is placed in a design, the cell origin will lie on the data point entered to position it.

- Click the Create button in Cell Library dialog box.

- Input the name of cell. Repeat above action to create other cells.



Figure 7: 3D visualization of rectangular pipes

\section{Implementation in Oracle Spatial and Microstation}

The concept for 3D visualisation of pipelines is tested in Oracle Spatial and Microstation. The test is performed on two data sets (from China and 
Netherlands). The data for pipelines are organised in SDO_Geometry, the Object-Relational model of Oracle Spatial. Discussion and examples regarding the SDO_Geometry model are available elsewhere in the literature (e.g. Zlatanova and Stoter, 2006, Zlatanova et al 2002).

The data sets used were prepared (as described in Section 3) to include the central line with its $\mathrm{x}, \mathrm{y}, \mathrm{z}$ - coordinates, terrain height (or depth, zt), diameter of pipe (d), width and height of ditch $(d, h)$. The description of a pipe network can be therefore seen as a function of six parameters per pipe segment, i.e. $\mathrm{P}=\mathrm{F}(\mathrm{x}, \mathrm{y}, \mathrm{z}, \mathrm{zt}, \mathrm{d}, \mathrm{h})$

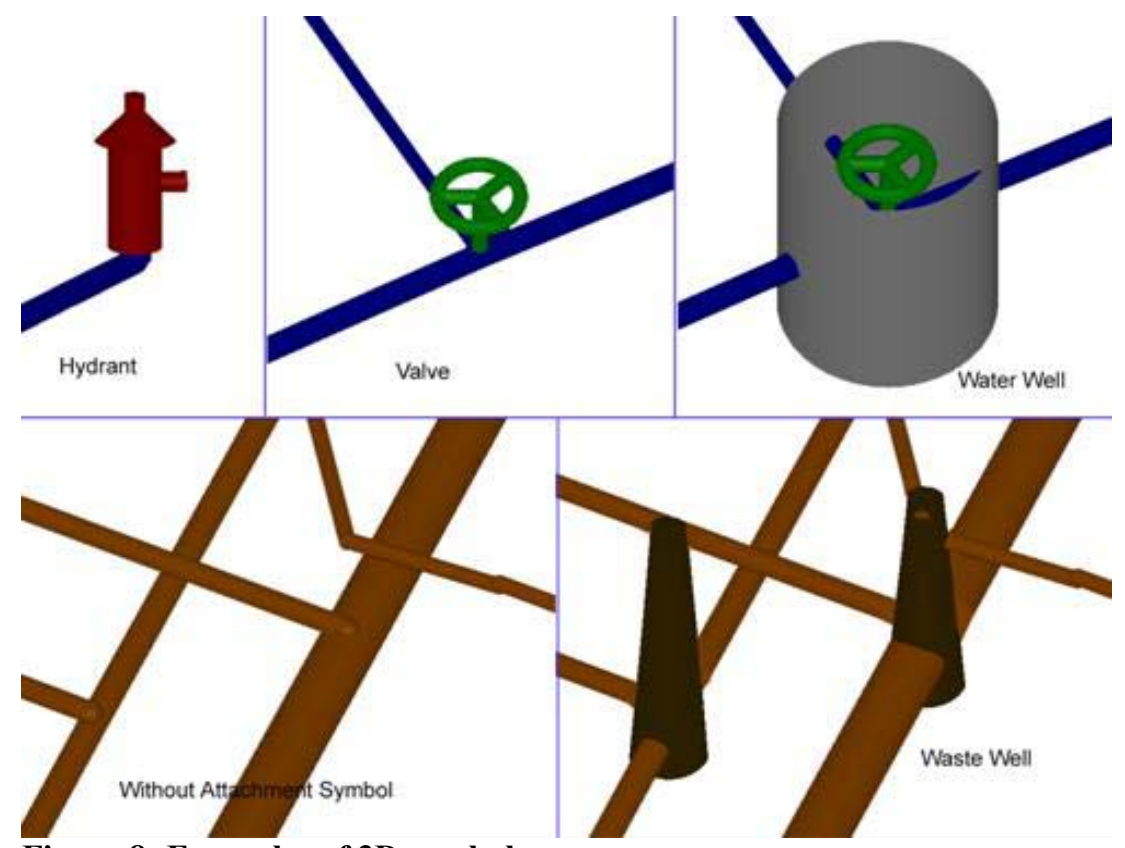

Figure 8: Examples of 3D symbols

SDO_Geometry supports only 4D coordinates. This means besides the coordinate $(\mathrm{x}, \mathrm{y}, \mathrm{z})$, only one more parameter can be included in the SDO_Geometry object. Since the terrain height is the most important value to obtain the depth, we have included it into SDO_Geometry as the fourth coordinate. It is used also for preparing vertical profile and calculating insertion points within pipelines. The advantage of this representation, i.e. terrain height instead of depth value, is that pipes above the ground can be also incorporated in the model. For example, a pipeline is under ground if (zt-z) is positive, otherwise, the pipeline is above ground.

The information about the pipelines is stored in two tables, i.e. 'line table' and 'point table'. The content of the line table follows: 
LINE TABLE

\section{Column Name}

mslink

start id

end $\overline{i d}$

MATTERIAL

diameter

tall

Pressure

Cabnum

Sumhole

Usedhole

bdate

lshape

segment, 4D)

Datatype
NUMBER(8)
NUMBER(8)
NUMBER(8)
VARCHAR(8)
NUMBER(6,3)
NUMBER(6,3)
VARCHAR(8)
NUMBER
NUMBER
NUMBER
VARCHAR(7)
GEOMETRY

Datatype

NUMBER(8)

NUMBER(8)

$\operatorname{VARCHAR}(8)$

NUMBER(6,3)

VARCHAR(8)

NUMBER

NUMBER
VARCHAR(7)

SDO_GEOMETRY

\author{
Description \\ PRIMARY KEY \\ Start Node \\ End Node \\ Material \\ Diameter (width of ditch) \\ Height of ditch \\ pressure (gas, electricity) \\ Cable number (electricity) \\ Total holes (telecom) \\ Used holes (telecom) \\ Build date \\ Gtype $=4002$ (pipeline
}

Clearly, the data contained in the line table is sufficient to visualize the pipelines in 3D and represent their location (both with respect to other networks and the terrain). However, some points contain attribute information, which cannot be assigned to the point if it is not a separate SDO_Geometry data type. Therefore those points that have specific attribute information are organized in a separate table. Practically, all the end points of a pipeline are represented by a 3D symbol and therefore the coordinates $(\mathrm{x}, \mathrm{y}, \mathrm{z})$ are still sorted in point table. Additional information that helps to create the 3D symbol can be height of ground; height of pipelines bottom and azimuth is also included.

\begin{tabular}{|c|c|c|}
\hline \multicolumn{3}{|l|}{ POINT TABLE } \\
\hline Column Name & Datatype & Description \\
\hline Mslink & NUMBER & ID of point \\
\hline Component & VARCHAR2(6) & Attachment or Node \\
\hline Top_h & NUMBER $(8,3)$ & Height/depth relative to ground \\
\hline Bot_h & $\operatorname{NUMBER}(8,3)$ & Bottom depth of pipeline \\
\hline Azimuth & number $(8,6)$ & Direction of symbol \\
\hline Pshape & Geometry & $\mathrm{x}, \mathrm{y}, \mathrm{z}$ coordinates \\
\hline
\end{tabular}

The Oracle Spatial data types can be readily accessed and visualised in Microstation, however, they would be visualised as they are stored in the database, i.e. as simple lines and points. Therefore, several new programs had to be developed within Microsation programming environment fo the visualisation on the fly. There are several development environments, such 
as MDL (Microstation Development Language), JMDL (Java edition of MDL) and VBA (Visual Basic for Applications) available in Microstation. We have selected JMDL.

Although JMDL is extensions of java in Microstation, there is a slight difference between JMDL and java. For example, JMDL supports the Boolean data type and all of the Java integral and floating-point types. In addition, JMDL supports unsigned types. Java operates on only two types: primitive types and reference types. JMDL operates on a third type, the complex type. The JMDL complex types are sort of structures and static Carrays, both of which are JMDL extensions. The Java implementation included in JMDL is completely standard. In the initial releases of Microstation/J, JMDL can be used to run existing Java applications, while standard Java interpreters are not be able to interpret JMDL byte code. This is due to the built-in extensions made to the JMDL. Java can also be used to develop JMDL applications that access Microstation via the DGN Package.

The DGN Package is a set of classes that allow Java/JMDL programmers to query and manipulate design (DGN) files. The methods contained in the DGN Package classes are not simply MDL functions wrapped up into JMDL methods. It is better to have classes that take advantage of the techniques that an object-oriented language provides like inheritance and message passing between objects. These classes are in the com.bentley.dgn package, such as ConeElemnt, ShapeElement, CellElement, etc. Further implementation details can be found in $\mathrm{Du}, 2005$.

Table 1: Number of records in the original data, 3D representation and using SDO Geometry

\begin{tabular}{|l|l|l|l|l|l|l|}
\hline \multirow{2}{*}{ Pipelines Type } & \multicolumn{3}{|l|}{$\begin{array}{l}\text { Original data } \\
\text { (RDBMS) }\end{array}$} & \multicolumn{2}{l|}{$\begin{array}{l}\text { Pipelines data } \\
(3 D)\end{array}$} & $\begin{array}{l}\text { OS } \\
\text { (SDO_geometry) }\end{array}$ \\
\cline { 2 - 8 } & Point & Segment & Point & Segment & Point & Line \\
\hline Communication & 788 & 768 & 968 & 917 & 422 & 407 \\
\hline Electricity & 1056 & 1046 & 1095 & 1071 & 383 & 513 \\
\hline Gas & 1937 & 1917 & 1939 & 1919 & 758 & 1293 \\
\hline Waste & 1241 & 1218 & 1592 & 1413 & 1174 & 386 \\
\hline Water & 4061 & 4076 & 4084 & 4089 & 1868 & 2260 \\
\hline
\end{tabular}

Tests are performed with several pipeline networks (Table 1). The table indicates also the number of records in the original data sets (only horizontal segments), the extended with vertical elements data set and the organisation using SDO_Geometry data type. The numbers in the second column illustrate the increased number of records due to the newly introduced vertical segments. The third column contains the number of records after us- 
ing the Oracle Spatial data type LINE. Clearly, the number of records is significantly reduced.

Additionally, 3D building data and terrain data are organized in the same DBMS following 3D data organizations approaches described in Zlatanova and Stoter 2006. While the pipeline data are displayed using the developed programs, the building and surface data are extracted from the database using the standard means of Bentley Geographics (e.g. Spatial Viewer).

Figure 9 portrays some of the obtained results. For clarity the terrain surface is not visualized in the snapshot.

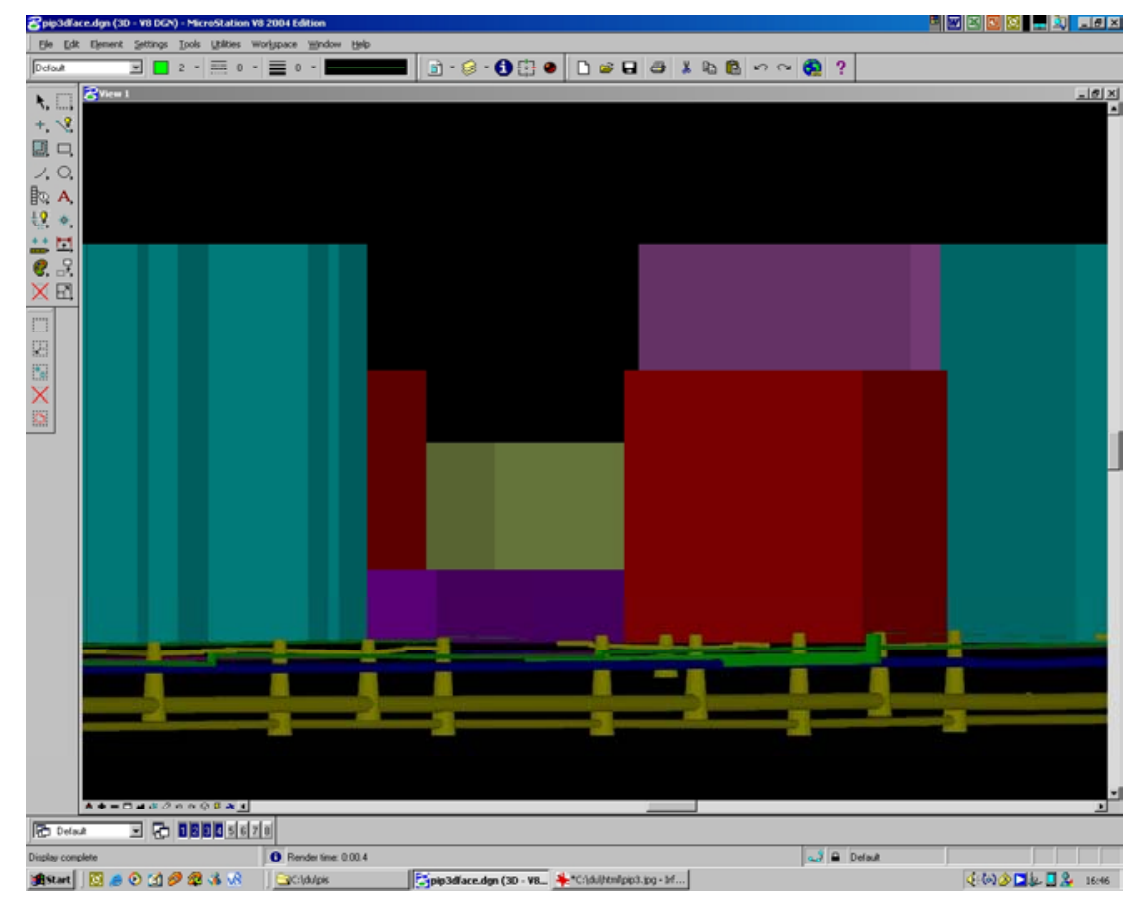

Figure 9: Examples of 3D visualization of pipelines

\section{Conclusions and further research}

This paper presented an approach for data organisation and 3D visualisation on the fly. All the experiments and test have clearly shown that 3D visualization of pipelines is much more appealing compared to $2 \mathrm{D}$ visualization. Relationships between pipes and other objects (buildings, road, surface data) are well visible in the 3D scene. The entire pipeline network is also much better represented and can be visually investigated. The inclu- 
sion of 3D symbols to show pipeline attachments (e.g. valves, hydrants, wells) helps to provide additional information on the particular pipelines, including its function, direction of flow, connectivity etc. Besides the improved visualisation, the reported approach has the following advantages:

The existing pipelines and cable data sets do not need to be changed besides for the creation of vertical segments (which can be automated and checked for consistency). Clearly, if existing pipeline networks do not have $\mathrm{z}$ coordinate, additional measurements to determine $\mathrm{z}$-coordinate (depth) are unavoidable.

Having preserved the original centre lines (stored in SDO_Geometry), editing of pipelines remains the same. If one or more segments have to be modified, the user can extract from DBMS only the centre lines and apply the usual procedure for editing using the preferred software, i.e. CAD or GIS.

The DBMS storage of pipelines allows for performing of spatial analysis, within the network and between other data sets (e.g. cadastre parcels), supposedly other data sets are also available in the DBMS. As mentioned elsewhere, the spatial functions and operations in Oracle Spatial are currently only $2 \mathrm{D}$ but operations accept the $3 \mathrm{D} / 4 \mathrm{D}$ coordinates. This means that a large number of queries such as 'which pipelines go under parcel 11' or 'which telecom cables are within 100 meter from my house', still can be performed.

The use of Oracle Spatial data type LINE has lead to a reduction of the number records compared to the original data sets.

Although the implementation is completed for Oracle Spatial and Microstation, the developed algorithms can be easily adapted for any frontend and therefore readily used for other combinations of DBMS and CAD/GIS software.

The selected system architecture, i.e. DBMS for storage of utility networks and front-end (CAD or GIS) for visualisation and editing, can be considered by many local and national authorities as a promising option for a centralised registering. The spatial schema in the DBMS can be tuned with respect to the legislation of a particular country.

Despite the promising results still various questions have to be investigated. Some specific functions for pipelines, i.e. analysis of section, profiles and intersections, as well as analysis of probably malfunctioning pipes and connections, have to be developed under the software used as a front-end. Additional program developments are desirable to refine the 3D visualization, such as torus, rectangular cylinder, sphere. A further study is needed to investigate which functions are worthy to implement at DBMS level to manage efficient and flexible different networks. 


\section{Acknowledgements}

The authors express their gratitude to the China Scholarship Council, Bentley Inc. and GDMC, TUDelft for making these developments possible.

\section{References}

Breunig, M. and S. Zlatanova, 2006, 3D Geo-DBMS, Chapter 4 in S. Zlatanova\&D. Prosperi (Eds.) Large-scale 3D Data Integration: challengies and opportunities, Taylor\&Francis, A CRC press book, pp. 88-113

Chong SC, 2006, Registration of Wayleave (cable and pipeline) into the Dutch cadastre, Case study report, available at http://www.gdmc.nl/publications, 56 p.

$\mathrm{Du}, \mathrm{Y} ., 2005,3 \mathrm{D}$ visualization of urban pipelines, Case study report, available at http://www.gdmc.nl/publications, $44 \mathrm{p}$.

He, J., L. Li and M. Deng, 2002, The design, of urban underground pipe line GIS, based on UML flexibility software developing model. IAPRS, Vol. XXXIV, PART2, Com II, Xi'an, Aug.20-23, China, pp. 169-170

Oosterom, P. van, J. Stoter, W. Quak and S. Zlatanova, 2002, The balance between geometry and topology, in: Advances in Spatial Data Handling, 10th International Symposium on Spatial Data Handling, D.Richardson and P.van Oosterom (Eds.), Springer-Verlag, Berlin, pp. 209-224

Peninga, F. and P van OOsterom, 2006, Kabel en leidingnetwerken in de kadastrale registratie, GISt rapport No 42, available at http://www.gdmc.nl/publications, 36 p. (in Dutch)

Peng, W., X. She, H. Xue and W. Znang, 2002, Integrating modeling for profile analysis of urban underground pipelines, based on 3D GIS, IAPRS, Vol XXXIV, PART2, Com. II, Xi'an, Aug.20-23, China, pp. 379-382

Roberts, G. et al, 2002. Look Beneath the Surface with Augmented reality, GPS World, February, pp. 14-20.

Yong, Y. 2003, Research On 3D Visualization Of Underground Pipeline, Transaction of Wuhan University, edition of science information, June, 2003

Zlatanova, S. 2004, Workshop on 3D city modelling, UDMS 2004, textbook, available at http://www.gdmc.nl/publications, $52 \mathrm{p}$

Zlatanova, S. and J. Stoter, 2006, The role of DBMS in the new generation GIS architecture, Chapter 8 in S.Rana\&J. Sharma (Eds.) Frontiers of Geographic Information Technology, Springer, pp. 155-180

Zlatanova, S. A.A. Rahman and M.Pilouk, 2002, Trends in 3D GIS development in: Journal of Geospatial Engineering, Vol.4, No.2, pp. 1-10 